\title{
STOCHASTIC MODELS FOR INTERFERENCE BETWEEN SEARCHING INSECT PARASITES
}

\author{
PHIL DIAMOND 1
}

(Received 24 March 1987; revised 1 June 1988)

\begin{abstract}
Competition between a finite number of searching insect parasites is modelled by differential equations and birth-death processes. In the one species case of intraspecific competition, the deterministic equilibrium is globally stable and, for large populations, approximates the mean of the stationary distribution of the process. For two species, both inter- and intraspecific competition occurs and the deterministic equilibrium is globally stable. When the birth-death process is reversible, it is shown that the mean of the stationary distribution is approximated by the equilibrium. Confluent hypergeometric functions of two variables are important to the theory.
\end{abstract}

\section{Introduction}

Insect parasites (more strictly, parasitoids) are entomophagous insects. They seek out certain states (egg, larvae) of an insect host and oviposit one or more eggs on or into the host. Typically, these eggs hatch and the parasitic larvae devour their host, pupate and the cycle begins anew.

Much of the behaviour of the actively searching parasitoids is apparently determined by functional responses to the host and other members of their own species, usually in a "patchy" environment of host dispersion ([5] and references therein, [12]). Various mechanisms of mutual interference which can reduce the available searching time, with a consequent effect upon searching efficiency, have been suggested. Rogers and Hassell [13] have pointed out that any mutual response among parasitoids raises the possibility of interference. They document observations where traces, left by preceding parasitoids, reduced the available searching time. The overall effect is that such mutual interference gives the

${ }^{1}$ Mathematics Department, University of Queensland, St. Lucia, Queensland 4067, Australia. (C) Copyright Australian Mathematical Society 1989, Serial-fee code 0334-2700/89 
appearance of a phenomenological decrease in the efficiency of the parasites, and the authors supply an elegantly simple static model to describe the effect.

Less is known about interspecific interference between different species of parasitoids. It has been observed that traces left by one species can inhibit another, although the response need not be reciprocal [4], [7]. Actual physical combat, in competition for hosts, has also been reported. It would seem very likely that either of these mechanisms reduces the time available for seeking out and parasitising hosts.

This note addresses these interference effects by modelling parasitic responses by deterministic and stochastic processes in which parasitoids can assume either active or passive states. The passive state corresponds to individual searching time lost, whereas the active state represents utilisation of actual searching time. This is a finite state, continuous time birth-death process with linear birth rate, quadratic death rate and reflecting barriers. The different degree of the rates causes some technical problems, and the techniques of neither [8] nor [11] seem applicable. Also, the rates are not density-dependent in the sense of Kurtz [10], and consequently his conditions for approximation by an underlying deterministic model are not satisfied. Instead, we show a weaker result: that the mean of the stationary process is asymptotic (for large population size) to the globally stable equilibrium point of a corresponding system of differential equations. For the one-species model case of intraspecific interference this is always true, and holds for the two-species model provided the process is reversible. The results differ from [2] in so far as all death rates are here quadratic and the method of partial differential-difference equations does not resolve the problem.

\section{Single species}

Assume that there are $N$ parasites, $P$ of which are actively searching and $P_{w}$ passive, indulging in no search for hosts: $N=P+P_{w}$. Suppose that a passive insect resumes activity after a waiting time. In the deterministic case model, this is taken as a constant $T$ over the population for all time. For the stochastic model, this waiting time is assumed to have an exponential distribution, of mean $T$. A parasitoid in the active state will become passive only if it encounters another, either through direct perception of another active parasite, or discovery of some trace such as a previously parasitised host.

Make the approximation that the encounter rate in the time interval $(t, t+d t)$, per active parasite, is proportional to $(P-1) d t+o(d t)$, and thus the chance of one parasite becoming passive is proportional to $P(P-1) d t+o(d t)$. This is perhaps a good approximation in the first type of encounter but, depending upon the duration for which a parasitised host carries some distinguishing feature 
which searching parasitoids can recognise, is perhaps not quite as good for the second type.

Consider first the deterministic case. Suppose that $P(t)$ is the number of actives at time $t$, and that $N$ is large enough for $P(t)$ to be considered as continuously differentiable, and $P(0)=N$. The dynamics of the interaction are assumed to be as in the equilibrium case: no lag is considered, and there are no terms in $P(t-T)$. Thus, for the encounter rate $b>0$,

$$
\begin{aligned}
& P(t+d t)-P(t)=((N-P(t)) / T-b P(t)(P(t)-1)) d t+o(d t), \\
& P^{\prime}(t)=(N-P) / T-b P(P-1) .
\end{aligned}
$$

It follows that $\lim _{t \rightarrow \infty} P(t)=\pi_{+}$, where $\pi_{+}$is the positive zero of $\phi(u)=b u^{2}+u(1 / T-b)-N / T$. For, write $\phi(u)=b\left(u-\pi_{+}\right)\left(u-\pi_{-}\right)$and let $V(u)=\left(u-\pi_{+}\right) \phi(u) / b$. Then $V(u)$ is positive definite in $[0, \infty) \backslash\left\{\pi_{+}\right\}=I$, and

$$
\begin{aligned}
V^{\prime}(P) & =P^{\prime} \phi(P) / b+\left(P-\pi_{+}\right) \phi^{\prime}(P) P / b \\
& =-\phi(P)\left(2 \phi(P)+\left(P-\pi_{+}\right)^{2}\right)<0 .
\end{aligned}
$$

So $V(u)$ is a strong Liapunov function on $I$ and the equilibrium $\pi_{+}$is asymptotically stable. Finally, observe that

$N^{-1} \pi_{+}=N^{-1}\left(-1+b T+\left((1-b T)^{2}+4 b T N\right)^{1 / 2}\right) / 2 b T=(b T N)^{-1 / 2}+O\left(N^{-1}\right)$

for large $N$.

Now suppose that the number of active parasites is governed by a birth-death process: in $(t, t+d t)$,

$$
\begin{aligned}
& \operatorname{Pr}\{n-1 \rightarrow n\}=(N-(n-1)) d t / T+o(d t), \\
& \operatorname{Pr}\{n+1 \rightarrow n\}=b n(n+1) d t+o(d t)
\end{aligned}
$$

let $p_{n}(t)=\operatorname{Pr}\{P(t)=n\}$. The assumptions concerning the way encounters occur preclude the possibility of a $1 \rightarrow 0$ transition, so $p_{0}(t)=0$ for all $t$, and further suppose that $p_{N}(0)=1$. Using the generating function $G(x, t)=\sum_{n=1}^{N} p_{n}(t) x^{n}$, we have

$$
\partial G / \partial t=(x-1) N G / T+T^{-1} x(1-x) \partial G / \partial x+b x(1-x) \partial^{2} G / \partial x^{2} .
$$

Look for separable solutions of the form $y(x) g(t)$, so that

$$
G(x, t)=\sum_{k} y_{k}(x) g_{k}(t)
$$

Then,

$$
g^{\prime} / g=(x-1) N / T+x(1-x) y^{\prime} / T y+b x(1-x) y^{\prime \prime} / y=-\lambda .
$$

Clearly $G$ is a sum of terms $e^{-\lambda t} y(x)$, where $\lambda$ is such that the equation

$$
y^{\prime \prime} b x(1-x)+y^{\prime} x(1-x) / T+(\lambda+(x-1) N / T) y=0
$$


has polynomial solutions of degree no greater than $N$. Amongst the eigenvalues are $\lambda_{0}=0$, which corresponds to the stationary distribution, and $\lambda_{N}=N / T$. Only the generating function of the stationary distribution is relevant to the remainder of the discussion. For $\lambda=0$, put $y=x u, x=-b T z$ in (3) to obtain $z d^{2} u / d z^{2}+(2-z) d u / d z+(N-1) u=0$. Recall the associated Laguerre polynomials ([3], vol. $2, \S 10.12$, although there are other related definitions), $L_{n}^{\alpha}(z)=$ $\sum_{m=0}^{\infty}\left(\begin{array}{c}n+\alpha \\ n-m\end{array}\right)(-z)^{m} / m !$, which satisfy $z d^{2} u / d z^{2}+(\alpha+1-z) d u / d z+n u=0$. Thus $u(z)=L_{N-1}^{1}(z)$ and $y(x)=(-x / b T) L_{N-1}^{1}(-x / b T)$. This must be normalised to act as a probability generating function, and then the stationary probabilities $p_{n}=\lim _{t \rightarrow \infty} p_{n}(t), n=1,2, \ldots, N$ take the form $p_{n}=a_{n} \theta^{n} / f(\theta)$, $n=1,2, \ldots, N, \theta=1 / b T$, where $f(\theta)=\sum_{n=1}^{N} a_{n} \theta^{n}=\theta L_{N-1}^{1}(-\theta)$. The factorial moments are given by $\mu_{(r)}=\theta^{r} f^{(r)}(\theta) / f(\theta)$. Recall ([3], vol. 2, $\S 10.12)$ that $d\left(L_{n}^{\alpha}(\theta)\right) / d \theta=-L_{n-1}^{\alpha+1}(\theta)$, and that $\theta L_{n}^{\alpha+1}(\theta)=(n+\alpha+1) L_{n}^{\alpha}(\theta)-$ $(n+1) L_{n+1}^{\alpha}(\theta)$. It follows that the mean of the stationary distribution is $\mu=$ $N\left(1-L_{N-2}^{1}(-1 / b T) / L_{N-1}^{1}(-1 / b T)\right)$, and the variance $\sigma^{2}=\mu-\mu^{2}+$ $N(N-1)\left(1-\left(2 L_{N-2}^{1}(-1 / b T)-L_{N-3}^{1}(-1 / b T)\right) / L_{N-1}^{1}(-1 / b T)\right)$.

Proposition 1. For large $N$, the mean searching efficiency of the stationary process is asymptotic to $N^{-1} \pi_{+}$of (2).

ProOF. Begin with the known recurrence

$$
(n+1) L_{n+1}^{\alpha}(\theta)-(2 n+1+\alpha-\theta) L_{n}^{\alpha}(\theta)+(n+\alpha) L_{n-1}^{\alpha}(\theta)=0 .
$$

Write $q_{n}(\theta)=L_{n}^{1}(\theta) / L_{n-1}^{1}(\theta)$ in this equation to obtain

$$
q_{n+1}(\theta)=2-\theta /(n+1)-1 / q_{n}(\theta)
$$

Now the expression for the mean order reads $\mu=N\left(1-1 / q_{N-1}(-1 / b T)\right)$, and the mean searching efficiency is the average number of active parasites per head of species population $\mu / N$. A rigorous argument along the lines of [15] pp. 223-225 justifies substituting $q_{n}(-\theta)=1+\sum_{k \geq 1} c_{k} n^{-k / 2}$ in (4), from which $q_{n}(-\theta)=1+\theta^{1 / 2} n^{-1 / 2}+n^{-1} / 4+O\left(n^{-3 / 2}\right)$ and so, as $n \rightarrow \infty, 1 / q_{n}(-\theta)=$ $1-\theta^{1 / 2} n^{-1 / 2}+O\left(n^{-1}\right)$, whence $\mu / N=(b T N)^{-1 / 2}+O\left(N^{-1}\right) \sim \pi_{+} / N$.

NOTES. 1. The mean and variance of this distribution are slightly unusual in being of order $N^{1 / 2}$, whereas it is commonly expected by population biologists that in natural populations these occur as $O(N)$ [12].

2. If only visual or physical contact be assumed, and that both parasites involved in the interaction then become passive, $p_{0}(t)$ is no longer zero because of the occurrence of $2 \rightarrow 0$ transitions. A similar analysis proceeds via confluent hypergeometric polynomials. The mechanism produces much the same effect upon searching efficiency as if one individual in the first model were to remain passive for time $2 T$. 
3. The stationary process, in this one-species case, is reversible. This is not automatically so in the two-species model of Section 4 . There, reversibility translates as a significant biological interaction between the species; see Lemma 1 of that section.

\section{Two species: deterministic interaction}

Suppose now that there are two distinct species of parasitoids: $N$ of type 1 , of which $P$ are actively searching, and $M$ of type 2 , of which $Q$ are active. Further, that passive insects of type 1 become active again after time $T_{1}$, those of type 2 after time $T_{2}$ and that these are constants for the populations over all time and independent of whether passivity is induced by an intraspecific or an interspecific interaction. Parasites are assumed to pass from the active to passive state only after encountering directly, or detecting some trace of, another active parasite of either species. Available evidence suggests that, by and large, some account should be taken of different encounter rates for intra- and interspecific interactions, although this is not always the case [4], [6], [7]. For $i, j=1,2$, denote by $b_{i j}>0$ the encounter rate of species type $i$ on type $j$, using the convention that $b_{\imath i}$ are intraspecific while $b_{\imath j}, i \neq j$, are interspecific rates.

The differential equations governing the number of active parasites $P(t), Q(t)$ at time $t$ are then

$$
\begin{aligned}
& d P / d t=(N-P) / T_{1}-b_{11} P(P-1)-b_{12} P Q, \\
& d Q / d t=(M-Q) / T_{2}-b_{22} Q(Q-1)-b_{21} Q P .
\end{aligned}
$$

Let $\eta(u)=(N-u) / T_{1}-b_{11} u(u-1), \psi(u)=(M-u) / T_{2}-b_{22} u(u-1)$, and let $\pi_{+}, \rho_{+}$be the positive zeros of $\eta(u), \psi$ respectively. The $d P / d t=0$ isocline is $Q=\eta(P) / b_{12} P=\Phi_{1}(P)$, and the $d Q / d t=0$ isocline is $P=\psi(Q) / b_{21} Q=$ $\Psi(Q)$. It is easy to see that $\Phi_{1}^{\prime}(P)=-\left(N+b_{11} T_{1} P^{2}\right) /\left(b_{12} T_{1} P^{2}\right)<0$ in $0<P<$ $\infty$, and that $Q=\Phi_{1}(P)$ crosses the $P$-axis at $P=\pi_{+}$and has the $Q$-axis as a vertical asymptote as $P \rightarrow 0+$. Similarly, $\Psi^{\prime}(Q)=-\left(M+b_{22} T_{2} Q^{2}\right) /\left(b_{21} T_{2} Q^{2}\right)<0$ in $0<Q<\infty$. This defines $Q=\Phi_{2}(P)=\Psi^{-1}(P)$, which crosses the $Q$-axis at $Q=\rho_{+}$and is horizontally asymptotic to the $P$-axis, $P \rightarrow \infty$ as $Q \rightarrow 0+$, and $\Phi_{2}^{\prime}(P)=1 / \Psi^{\prime}(Q)$. See the figure for details.

PROPOSITION 2. Let $b_{12} b_{21} \leq b_{11} b_{22}$. Then (5) has a unique equilibrium point $\left(P^{*}, Q^{*}\right)$ in the positive quadrant for all integers $N, M$, which is moreover asymptotically stable for all initial conditions in the positive quadrant.

PROOF. If the isoclines meet at a unique point in the positive quadrant, with the geometry described above, then elementary considerations show this point 


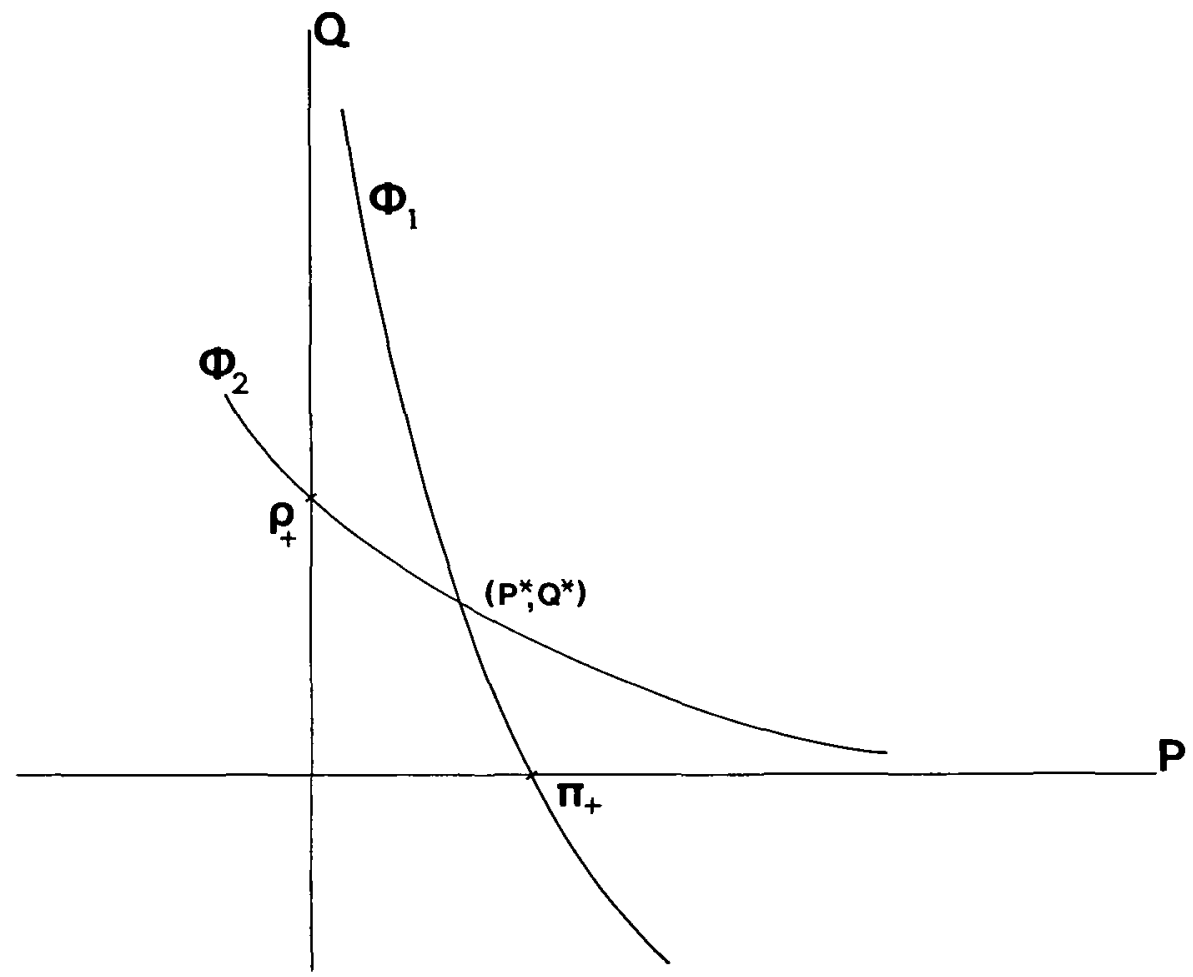

FIgurE. The isoclines of the DE (5), with unique equilibrium point $\left(P^{*}, Q^{*}\right)$ in the positive quadrant.

to be asymptotically stable. Since $0 \leq \Phi_{1}(P)<\infty$ on $\left(0, \pi_{+}\right]$, and $\Phi_{2}(P)>0$ there, $\varsigma(P)=\Phi_{1}(P)-\Phi_{2}(P)$ has at least one zero on $\left(0, \pi_{+}\right]$. To show this is unique, it is sufficient to show $\zeta^{\prime}(P)<0$ on $\left(0, \pi_{+}\right]$. But for $0<P<\pi_{+}$, $0<Q<\rho_{+}, \varsigma^{\prime}(P)=\Phi_{1}^{\prime}(P)-1 / \Psi^{\prime}(Q)$, and

$$
\begin{aligned}
\varsigma^{\prime}(P)\left(M+b_{22} T_{2} \rho_{+}^{2}\right) b_{12} T_{1} \pi_{+}^{2}= & P^{2} Q^{2}\left(b_{12} b_{21}-b_{11} b_{12}\right)-b_{11} T_{1} M P^{2} \\
& -b_{22} T_{2} N Q^{2}-M N<0,
\end{aligned}
$$

provided $b_{12} b_{21} \leq b_{11} b_{22}$.

If $b_{12} b_{21}=b_{11} b_{22}$, the asymptotic form of $P^{*}, Q^{*}(M, N$ large) is relatively simple. Write $\beta_{1}^{-2}=b_{11} T_{1}, \beta_{2}^{-2}=b_{22} T_{2}$. Direct substitution in the isocline equations $Q=\Phi_{1}(P), P=\Psi(Q)$ verifies

PROPOSITION 3. Let $b_{12} b_{21}=b_{11} b_{22}$, suppose that $b_{11} / b_{12}=\sigma$ and write $f(N, M)=\left(\beta_{1}^{2} N+\sigma^{2} \beta_{2}^{2} M\right)^{-1 / 2}$. Then

$$
\begin{aligned}
& P^{*}=\beta_{1}^{2} N f(N, M)+\left(1-\beta_{1}^{2}\right) / 2+O(f(N, M)), \\
& Q^{*}=\sigma \beta_{2}^{2} M f(N, M)+\left(1-\beta_{2}^{2}\right) / 2+O(f(N, M)) .
\end{aligned}
$$




\section{Two species: birth-death process}

Now suppose that the numbers of active parasites are governed by a birthdeath process with rates $b_{i j}$ as defined in the previous section. In $(t, t+d t)$, up to $o(d t)$,

$$
\begin{aligned}
& \operatorname{Pr}\{(n-1, m) \rightarrow(n, m)\}=(N-(n-1)) d t / T_{1}, \\
& \operatorname{Pr}\{(n, m-1) \rightarrow(n, m)\}=(M-(m-1)) d t / T_{2}, \\
& \operatorname{Pr}\{(n+1, m) \rightarrow(n, m)\}=\left(b_{11} n(n+1)+b_{12} m(n+1)\right) d t, \\
& \operatorname{Pr}\{(n, m+1) \rightarrow(n, m)\}=\left(b_{22} m(m+1)+b_{21}(m+1) n\right) d t .
\end{aligned}
$$

Let $p_{n m}(t)=\operatorname{Pr}\{P=n$ and $Q=m$ at time $t\}$. Observe that $p_{00}(t)=0$ and $p_{n m}(t)=0$ if $n>N$ or $m>M$, while the initial condition is assumed to be $p_{N M}(0)=1$ (i.e., all parasites commence in an active state). The generating function $H(x, y, t)=\sum_{m+n \geq 1} p_{n m}(t) x^{n} y^{m}$ thus has initial condition $H(x, y, 0)=x^{N} y^{M}$, and satisfies

$$
\begin{aligned}
H_{t}= & b_{11} x(1-x) H_{x x}+b_{22} y(1-y) H_{y y}+\left(b_{12} y(1-x)+b_{21} x(1-y)\right) H_{x y} \\
& +x(1-x) H_{x} / T_{1}+y(1-y) H_{y} / T_{2}-N(1-x) H / T_{1}-M(1-y) H / T_{2} \\
= & \mathscr{L}_{N M}(H) .
\end{aligned}
$$

On writing $H=U(t) V(x, y)$ there exists $\lambda_{\imath j}, i=0,1, \ldots, N, \quad j=0,1, \ldots$, $M-1\left(\lambda_{00}=0, \lambda_{N M}=-(N-1) / T_{1}-(M-1) / T_{2}\right)$, so that the solution may be expressed in the form

$$
H(x, y, t)=\sum_{i=0}^{N-1} \sum_{j=0}^{M-1} \exp \left(\lambda_{i j} t\right) V_{i j}(x, y) .
$$

Here $V_{\imath \jmath}$ is a 2-variable polynomial of bi-degree no greater than $(N, M)$, satisfying $\mathscr{L}_{N M}(V)=\lambda_{i j} V$. Let $p_{n m}=\lim _{t \rightarrow \infty} p_{n m}(t)$ be the limiting distribution, whose existence is guaranteed by the standard theory. Clearly the generating function for the stationary distribution corresponds to the eigenvalue $\lambda=0$, and so $\mathscr{L}_{N M}(V)=0$. Direct calculation shows that

$$
\begin{gathered}
\left(b_{11} n(n+1)+b_{12} m(n+1)\right) p_{n+1, m}+\left(b_{22} m(m+1)+b_{21} n(m+1)\right) p_{n, m+1} \\
-\left((N-n) / T_{1}+(M-m) / T_{2}+b_{11} n(n-1)\right. \\
\left.+b_{22} m(m-1)+\left(b_{12}+b_{21}\right) m n\right) p_{n m} \\
+(N-(n-1)) p_{n-1, m} / T_{1}+(M-(m-1)) p_{n, m-1} / T_{2}=0 .
\end{gathered}
$$

Now $p_{01}(M-1) / T_{2}-b_{12} p_{11}-2 b_{22} p_{02}=0$ expresses the balance for the $(0,1)$, $(1,1)$ and $(0,2)$ states at equilibrium, and $p_{10}(N-1) / T_{1}-b_{21} p_{11}-2 b_{11} p_{20}=0$ between that of the $(1,0),(1,1)$ and $(2,0)$ states. Thus equation $(6)$ may be 
split into two expressions, both equalling zero, and so

$$
\begin{gathered}
(n+1)\left(b_{11} n+b_{12} m\right) p_{n+1, m}+(m+1)\left(b_{21} n+b_{22} m\right) p_{n, m+1} \\
-\left((N-n) / T_{1}+(M-n) / T_{2}\right) p_{n m}=0
\end{gathered}
$$

LEMMA 1. The stationary distribution is reversible if and only if each species cannot distinguish between individuals of its own and the other species: $b_{11}=b_{12}$ and $b_{22}=b_{21}$.

PROOF. We have reversibility if and only if both

$$
\begin{aligned}
& (N-n) p_{n m} / T_{1}=\left(b_{11}(n+1) n+b_{12}(n+1) m\right) p_{n+1, m} \\
& (M-m) p_{n m} / T_{2}=\left(b_{22}(m+1) m+b_{21}(m+1) n\right) p_{n, m+1} .
\end{aligned}
$$

Passing from the $(n+1, m+1)$ state to $(n, m)$ by the two possible paths $(n+1, m+$ $1) \rightarrow(n, m+1) \rightarrow(n, m)$ and $(n+1, m+1) \rightarrow(n+1, m) \rightarrow(n, m)$ gives by (8) that $\left(b_{11} n+b_{12}(m+1)\right)\left(b_{22} m+b_{21} n\right)$ should agree with $\left(b_{11} n+b_{12} m\right)\left(b_{22} m+\right.$ $b_{21}(n+1)$ ) for all $n=1, \ldots, N, \quad m=1, \ldots, M$. That is, $b_{12} b_{22}=b_{12} b_{21}$ and $b_{21} b_{11}=b_{21} b_{12}$. Under the assumption that was made, that $b_{21}, b_{12}$ were nonzero, it must follow that $b_{11}=b_{12}$ and $b_{22}=b_{21}$.

In all that follows, the stationary distribution is supposed reversible. The main difference from the one-species case is

PROPOSITION 4. Denote by $V^{N, M}(x, y)$ the polynomial solution of $\mathscr{L}_{N M}(V)=$ 0 . Then $\left\{V^{N, M}\right\}$ cannot form a system of orthogonal polynomials in two variables.

PROOF. The operator $\mathscr{L}_{N M}$ does not satisfy the necessary and sufficient conditions of [9], Theorem 3.1.

PROPOSITION 5. The stationary probabilities of the reversible process are given by $p_{n m}=\theta_{1}^{n} \theta_{2}^{m} q_{n m} / V^{N, M}(1,1)$, where $\theta_{1}=-\beta_{1}^{2}, \theta_{2}=-\beta_{2}^{2}$ and $q_{n m}=$ $(-M)_{m-1}(-N)_{n-1} / m ! n !(m+n-1)$ !

PROOF. Follows from Lemma 1, (8) and verification that (7) is satisfied.

In Horn's list of hypergeometric functions of two variables $([3], \S 5.7 .1)$ is the function

$$
\Phi_{2}\left(\alpha, \alpha^{\prime}, \gamma, x, y\right)=\sum_{n, m=0}(\alpha)_{n}\left(\alpha^{\prime}\right)_{m} x^{n} y^{m} /\left(n ! m !(\gamma)_{n+m}\right)
$$

Observe that

$$
V^{N, M}(x, y)=\Phi_{2}\left(-N,-M, 1, x \theta_{1}, y \theta_{2}\right)-1
$$


is a terminating polynomial for positive integers $N, M$. The following properties of $\Phi_{2}$ can be found in [1] or are easily verified from comparing coefficients of $x^{n} y^{m}$ (the $x, y$ arguments and subscript have been suppressed):

$$
\begin{gathered}
\gamma \Phi\left(\alpha+1, \alpha^{\prime}, \gamma\right)-\gamma \Phi=x \Phi\left(\alpha+1, \alpha^{\prime}, \gamma+1\right) \\
\gamma \Phi\left(\alpha, \alpha^{\prime}+1, \gamma\right)-\gamma \Phi=y \Phi\left(\alpha, \alpha^{\prime}+1, \gamma+1\right) \\
\partial \Phi / \partial x=\alpha \Phi\left(\alpha+1, \alpha^{\prime}, \gamma+1\right) / \gamma, \quad \partial \Phi / \partial y=\alpha^{\prime} \Phi\left(\alpha, \alpha^{\prime}+1, \gamma+1\right) / \gamma \\
(\alpha-\gamma) y \Phi\left(\alpha-1, \alpha^{\prime}, \gamma\right)+\alpha^{\prime} x \Phi\left(\alpha, \alpha^{\prime}-1, \gamma\right)-\left(x y+(2 \alpha-\gamma) y+2 \alpha^{\prime} x\right) \Phi \\
+\alpha y \Phi\left(\alpha+1, \alpha^{\prime}, \gamma\right)+\alpha^{\prime} x \Phi\left(\alpha, \alpha^{\prime}+1, \gamma\right)=0 \\
\alpha y \Phi\left(\alpha-1, \alpha^{\prime}, \gamma\right)+\left(\alpha^{\prime}-\gamma\right) x \Phi\left(\alpha, \alpha^{\prime}-1, \gamma\right)-\left(x y+2 \alpha y+\left(2 \alpha^{\prime}-\gamma\right) x\right) \Phi \\
+\alpha y \Phi\left(\alpha+1, \alpha^{\prime}, \gamma\right)+\alpha^{\prime} x \Phi\left(\alpha, \alpha^{\prime}+1, \gamma\right)=0 .
\end{gathered}
$$

It follows from (13) that $\partial V^{N, M} / \partial x=-N \theta_{1} \Phi_{2}\left(-N+1,-M, 1, x \theta_{1}, y \theta_{2}\right)$, $\partial V^{N, M} / \partial y=-m \theta_{2} \Phi_{2}\left(-N,-M+1,1, x \theta_{1}, y \theta_{2}\right)$. Considering $p_{n m}$ as a power series distribution, the stationary mean number of actively searching parasites of types 1,2 respectively are

$$
\begin{aligned}
& \mu_{1}=-N \theta_{1} \Phi_{2}\left(-N+1,-M, 2, \theta_{1}, \theta_{2}\right) / V^{N, M}(1,1), \\
& \mu_{2}=-M \theta_{2} \Phi_{2}\left(-N,-M+1,2, \theta_{1}, \theta_{2}\right) / V^{N, M}(1,1) .
\end{aligned}
$$

Differentiating twice gives the covariance

$$
c_{12}=N M \theta_{1} \theta_{2} \Phi_{2}\left(-N+1,-M+1,3, \theta_{1}, \theta_{2}\right) / V^{N, M}(1,1)^{2}-\mu_{1} \mu_{2} .
$$

From (9), (10) and (13)

$$
\begin{aligned}
& \mu_{1}=N\left(1-V^{N+1, M}(1,1) / V^{N, M}(1,1),\right. \\
& \mu_{2}=M\left(1-V^{N, M+1}(1,1) / V^{N, M}(1,1)\right) .
\end{aligned}
$$

Proposition 6. As $N, M \rightarrow \infty$, in the notation of Proposition 3 with $\sigma=1$, $\mu_{1} \sim P^{*}$ and $\mu_{2} \sim Q^{*}$.

Proof. Write $f(N, M)=\left(\beta_{1}^{2} N+\beta_{2}^{2} M\right)^{-1 / 2}$ and let $v_{N}(M)=$ $\Phi_{2}\left(-N-1,-M, 1, \theta_{1}, \theta_{2}\right) / \Phi_{2}$, and $w_{M}(N)=\Phi_{2}\left(-N,-M-1,1, \theta_{1}, \theta_{2}\right) / \Phi_{2}$. It is sufficient to show that $v_{N}(M) \sim 1-\beta_{1}^{2} f(N, M), w_{M}(N) \sim 1-\beta_{2}^{2} f(N, M)$. Note that by dividing (12a) throughout by $\Phi$ and substituting the appropriate parameters, one obtains

$$
\begin{gathered}
(N+1) \beta_{2}^{2} / v_{N-1}(M)+M \beta_{1}^{2} / w_{M-1}(N)-\left(\beta_{1}^{2} \beta_{2}^{2}+(2 N+1) \beta_{2}^{2}+2 M \beta_{1}^{2}\right) \\
+N \beta_{2}^{2} v_{N}(M)+M \beta_{1}^{2} w_{M}(N)=0
\end{gathered}
$$


A similar expression $\left(12^{\prime} \mathrm{b}\right)$ results from performing the same operations on $(12 \mathrm{~b})$. Further, $f(N-1, M) \sim f(N, M)-\frac{1}{2} \beta_{1}^{2} f^{3}$ and $f(N, M-1) \sim f-\frac{1}{2} \beta_{2}^{2} f^{3}$. Substitution for $v_{N}, w_{M}$ in $\left(12^{\prime} \mathrm{a}\right),\left(12^{\prime} \mathrm{b}\right)$ gives asymptotic expressions as required, after a tedious calculation.

REMARK. It is possible to extend these interference models to any number $k$ of species, and stationary distributions will exist. Means and covariances are expressed in terms of confluent functions of $k$ variables $\Phi_{B}$, associated with the Lauricella functions $F_{D}[13]$ in much the same way as $\Phi_{2}$ is a confluence of $F_{1}$ [1].

\section{References}

[1] P. Appell and J. Kampé de Fériet, Fonctions hypergéomètriques et hypersphérıques (Gauthier-Villars, Paris, 1926).

[2] N. G. Becker, "A stochastic model for two interacting populations," J. Appl. Prob. 7 (1970), 544-564.

[3] A. Erdelyi et al., Higher Transcendental Functions (McGraw-Hill, New York, 1953-55).

[4] D. Force, "Competition among four Hymenopterous parasites of an endemic insect host," Ann. Ent. Soc. America 63 (1970), 1675-1688.

[5] M. P. Hassell, The dynamics of arthropod predator-prey systems (Princeton University Press, 1978).

[6] N. Hokyo and N. Kiritani, "Oviposition behaviour of two egg parasites, Asolcus Mitzukurii Ashmead and Telenomus Nakagawai Watanabe," Entomophagia 11 (1966), 191-201.

[7] N. Hokyo et al., "The effect of intra- and interspecific conditioning of host eggs on the ovipositional behaviour of two scalionid egg parasites of the Southern Green Stink Bug," Nezara Viridula L., Jap. J. Ecology 16 (1966), 67-71.

[8] S. Karlin and J. McGregor, "Linear growth and applications of special multidimensional Hahn polynomials," in Theory and applications of special functions (ed. R. A. Askey), (Academic Press, New York, 1975), 261-288.

[9] H. L. Krall and I. M. Scheffer, "Orthogonal polynomials in two variables," Ann. Mat. Pura. Appl. 76 (1967), 325-376.

[10] T. G. Kurtz, Approximation of population processes (SIAM, Philadelphia, 1981).

[11] J. Letessier and G. Valent, "The generating function method for quadratic asymptotically symmetric birth and death processes," SIAM J. Appl. Math. 44 (1984), 773-783.

[12] R. M. May, Stability and complexity in model ecosystems (2nd ed., Princeton University Press, 1974).

[13] W. R. Miller, Symmetry and separation of variables (Addison-Wesley, Reading, Mass., 1977).

[14] D. J. Rogers and M. P. Hassell, "General models for insect parasite searching behaviour: interference," J. Anim. Ecol. 43 (1974), 239-253.

[15] G. Szekeres, "Regular iteration of real and complex functions," Acta Math. Scand. 100 (1958), 203-258. 